\title{
Improved Beta-Cell Function and Altered Plasma Levels of GLP-1 During OMTT and Fasting Following Bariatric Surgery in Women with NGT
}

ISSN: 2578-0263

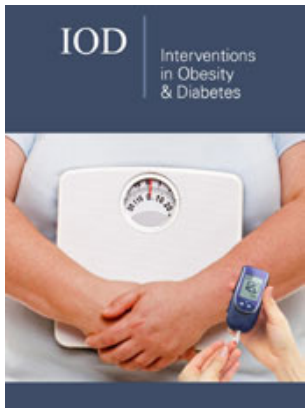

*Corresponding author: Camilla Krizhanovskii, Karolinska InstitutetSödertälje hospital, Department of Research, Lagmansvägen, Sweden

Submission: 漹 February 13, 2021

Published: March 16, 2021

Volume 5 - Issue 1

How to cite this article: Stelia Ntika, Sven Bringman, Per Lundquist, Linda Tracy, Camilla Krizhanovskii. Improved BetaCell Function and Altered Plasma Levels of GLP-1 During OMTT and Fasting Following Bariatric Surgery in Women with NGT. Interventions Obes Diabetes 5(1). IOD. 000604. 2021.

DOI: 10.31031/IOD.2021.05.000604

Copyright@ Camilla Krizhanovskii. This article is distributed under the terms of the Creative Commons Attribution 4.0 International License, which permits unrestricted use and redistribution provided that the original author and source are credited.

\author{
Stelia Ntika ${ }^{1,2}$, Sven Bringman ${ }^{3,4}$, Per Lundquist ${ }^{4}$, Linda Tracy $^{2}$ and Camilla \\ Krizhanovskiii, ${ }^{1, *}$ \\ ${ }^{1}$ Karolinska Institutet, Department of Molecular Medicine and Surgery, Sweden \\ ${ }^{2}$ Department of Research, Södertälje University Hospital, Sweden \\ ${ }^{3}$ Karolinska Institutet, Department of Clinical Sciences, Danderyd Hospital, Sweden \\ ${ }^{4}$ Department of Surgery, Södertälje University Hospital, Sweden
}

\begin{abstract}
Context: Bariatric surgery can be considered metabolic surgery, with important weight loss-independent effects on metabolism. Altered secretion of the intestinal hormone Glucagon-Like Peptide-1 (GLP1) may be an important factor in the metabolic success of bariatric surgery. GLP-1 is an important regulator of glucose metabolism, reducing glycemia by regulating insulin and glucagon secretion, slowing gastric emptying, and lowering the desire for food intake. A potentiating effect of bariatric surgery on postprandial GLP-1 secretion is well documented and contributes to the increased insulin secretion and sensitivity that precedes weight loss. However, studies on patients with Normal Glucose Tolerance (NGT) are relatively few, and a clear consensus is still lacking in terms of potential effects of bariatric surgery on fasting GLP-1 levels in obese subjects with NGT.
\end{abstract}

Objective: To investigate fasting and postprandial levels of GLP-1 in obese women with NGT before and after bariatric surgery.

Design: Plasma GLP-1 was quantified at fasting and during an Oral Meal Tolerance Test (OMTT) in female patients with NGT subjected to Vertical Sleeve Gastrectomy (VSG) or Roux-en-Y Gastric Bypass (RYGB), preoperatively and 3 months postoperatively. Insulin resistance was quantified using HOMA-IR, and beta cell function using the Oral Disposition Index (DIo).

Setting: This study was performed at Karolinska Institutet, Södertälje Hospital south of Stockholm, Sweden.

Patients and participants: Eleven female patients aged 18-65 years with a BMI $\geq 40 \mathrm{~kg} / \mathrm{m}^{2}$ and NGT were included. Exclusion criteria were T2D (based on the criteria of the American Diabetes Association (ADA) (2014)), prior GLP-1 based therapy or insulin therapy, and history of substance abuse (drugs or alcohol). Main outcome measures: Outcome measures were fasting and OMTT plasma levels of insulin and GLP-1. Results: Fasting GLP-1 levels were decreased 3 months postoperatively compared to baseline $(12.3 \pm 1.5$ vs. $20.1 \pm 3.4 \mathrm{pmol} / \mathrm{L}, \mathrm{p}=0.05$ ). Meal-stimulated GLP-1 secretion was significantly increased 3 months postoperatively in association with increased insulin sensitivity and enhanced beta cell function.

Conclusion: Bariatric surgery reduces fasting plasma GLP-1 levels, while significantly increasing mealstimulated plasma GLP-1 levels, in obese women with NGT.

Keywords: Bariatric surgery; Normal Glucose Tolerance (NGT); Obesity; BMI; Glucagon Like Peptide 1 (GLP-1)

Abbreviations: GLP-1: Glucagon-Like Peptide-1; NGT: Normal Glucose Tolerance; OMTT: Oral Meal Tolerance Test; VSG: Vertical Sleeve Gastrectomy; RYGB: Roux-en-Y Gastric Bypass; T2D: Type 2 Diabetes; IGT: Impaired Glucose Tolerance; ADA: American Diabetes Association

\section{Introduction}

Obesity is increasing worldwide, associated with the epidemic increase in Type 2 Diabetes (T2D), and thus constitutes a leading cause of global morbidity and mortality, placing an enormous burden on healthcare systems. Bariatric surgery is the best available treatment of severe obesity and has been associated with T2D remission [1,2]. Two of the most popular bariatric surgical procedures -- Vertical Sleeve Gastrectomy (VSG) and Roux-en-Y Gastric 
Bypass (RYGB) -- are sometimes referred to as metabolic surgeries because they affect metabolism by weight loss-independent mechanisms. Understanding the metabolic effects of bariatric surgery, and the mechanisms that mediate metabolic success and weight loss, is key to developing novel, less invasive and superior strategies to treat obesity and metabolic disease. Increased endogenous Glucagon-Like Peptide-1 (GLP-1) signaling has been considered a primary pathway leading to postsurgical weight loss and improvements in glucose metabolism following VSG and RYGB. GLP-1 is a gastrointestinal peptide hormone derived from tissue specific posttranslational processing of proglucagon and is one of the two incretin hormones increasing meal-stimulated insulin secretion. In addition, GLP- 1 stimulates $\beta$-cell proliferative and antiapoptotic pathways, exerts protective effects on cardiomyocytes, reduces insulin resistance, reduces glucagon concentrations by restoring hyperglycemic responses of the $\alpha$-cells, while also inhibiting gastric emptying, and food intake [3]. Attenuated GLP-1 responses to meals have been demonstrated in obese adolescents $[3,4]$. Other studies show no difference in fasting and stimulated GLP-1 between normal-weight and obese individuals [5]. Weight loss has been indicated to decrease [6], increase [4] or exert no effect on fasting and stimulated GLP-1 levels [7]. Increased postprandial GLP-1 secretion has repeatedly been shown following VSG as well as RYGB, with both total and active levels of GLP-1 increasing, and the intact hormone reaching nearly 10 times higher concentrations after RYGB and VSG [8]. Some studies have suggested that RYGB increases postprandial GLP-1 to a greater extent than VSG $[9,10]$. However, most studies demonstrate similar postprandial increases in active GLP-1 following both procedures [11-13]. Although many of these studies include patients with T2D or Impaired Glucose Tolerance (IGT), complicating the interpretation of the results, bariatric surgery is consistently associated with increased postprandial GLP-1 also among individuals with NGT. However, potential effects of bariatric surgery on fasting plasma GLP-1 levels in non-diabetic subjects remain elusive, and so also the effects of surgical procedure/potential gender differences. In a recent meta-analysis designed to assess fasting GLP-1 levels after RYGB, 15 studies were included and no significant difference between preoperative and postoperative fasting plasma GLP-1 levels was detected [14].

However, of the 15 studies included in this meta-analysis, half were performed on subjects with T2D or included subjects with T2D [10,15-20]. Five of the remaining studies analyzed only fasting plasma active GLP-1 7-36 [21-25], which may reflect differences in degradation and elimination rather than secretion. Due to the rapid intravascular degradation of GLP-1, it is essential to determine both the intact hormone and the metabolite for estimation of its rate of secretion. The three remaining studies included in this meta-analysis and assaying fasting total GLP-1 before and after bariatric surgery in NGT patients, showed no change in fasting plasma GLP-1 [26-28]. However, in a recent study, not included in this meta-analysis a significant increase in fasting plasma total GLP-1 was reported after bariatric surgery [29]. One weakness of all these studies, although similar in size to the present study (6-13 patients), is the lack of consideration for potential gender differences, and all studies report differences before and after RYGB. Although a number of studies have also been performed where fasting total plasma GLP-1 in postsurgical subjects have been compared to other postsurgical patient groups or other control groups [30-32], the effects of obesity and bariatric surgery on fasting GLP-1 levels in NGT subjects are thus still not conclusive, wherefore we aimed to assess the fasting and meal-stimulated plasma GLP-1 concentrations in female patients with NGT before and after bariatric surgery, independent of surgical procedure.

\section{Materials and Methods}

\section{Study population}

Non-diabetic female patients 18-65 years of age with a $\mathrm{BMI} \geq 40 \mathrm{~kg} / \mathrm{m}^{2}$ and NGT were included. All subjects were part of a larger clinical study at Södertälje Hospital (ISRCTN registry Identifier: ISRCTN11764561) and the Bypass Equipoise Sleeve Trial (BEST) (ClinicalTrials.gov Identifier: NCT02767505) and visited the obesity clinic at Södertälje Hospital between October 2016 and December 2018. Exclusion criteria were T2D (based on the criteria of the American Diabetes Association (ADA) (2014)), prior GLP-1 based therapy or insulin therapy, and history of substance abuse (drugs or alcohol). Written informed consent was obtained from all study subjects. Ethical permission was received from the Stockholm Regional Ethical Committee: (Dnr: 2015/795-31/2; approved: 2015-08-26). Up until the cutoff date of this preliminary report 15 women had been included in the study. Four of these patients dropped out before the postsurgical visit, leaving 11 women to be included in this report. However, based on the significant and interesting effect on fasting plasma GLP-1 and the similar size of previously reported studies, the data obtained are herein submitted as a preliminary report of the larger clinical trial. Glucose tolerance was defined according to World Health Organization criteria for plasma glucose and thus the definitions were: T2DM fasting glucose higher than $7.0 \mathrm{mM}$ and/or two-hour glucose higher than $11.1 \mathrm{mM}$, and NGT fasting glucose below $6.1 \mathrm{mM}$ and two-hour glucose below 7.8mM.

\section{Anthropometry}

Body weight was measured by a digital scale and height by a stadiometer. BMI was calculated as weight in kilograms divided by the square of the height in meters.

\section{Oral Meal Tolerance Test (OMTT)}

After a 12-hour overnight fast, subjects underwent a standard OMTT ingesting a meal replacement drink $200 \mathrm{ml}$ of Fresubin meal replacement drink (200kcal, carbohydrate $(27.2 \mathrm{~g})$, protein (7.6g), and fat (6.8g) (Fresenius Kabi Deutschland, Bad Homburg, Germany). The meal was ingested over a 10 -min period before and three months after surgery. Blood was sampled from a catheter in an antecubital vein at $-10,15,30,60$ and 120 minutes following meal intake (0 minutes). Blood samples for determination of GLP1 and insulin were collected in EDTA-containing vacutainer tubes (Becton Dickinson). Tubes were centrifuged for 10 minutes, and plasma stored at $-80 \mathrm{C}$ pending analyses. 


\section{Biochemical analyses}

Plasma glucose levels were determined using Contour XT test strips (Ascensia Diabetes Care). Plasma levels of GLP-1 were quantified by an absorbency sandwich ELISA-based assay (Merck, Millipore). The antibody used in this assay is specific to GLP-1 (736) and (9-36) and has no cross-reactivity with GLP-2, GIP, glucagon or oxyntomodulin. The limit of detection of the assay was $1.5 \mathrm{pM}$ and all concentrations measured were above this limit of detection. Plasma levels of insulin were quantified by ELISA (Mercodia). The insulin assay has a cross-reactivity with C-peptide and proinsulin of less than $0.01 \%$.

\section{Calculations and statistical analysis}

HOMA-IR was calculated as fasting insulin $(\mathrm{uIU} / \mathrm{mL})^{*}$ fasting glucose $(\mathrm{mg} / \mathrm{dL})$. The oral disposition index was used to estimate $\beta$-cell function before and after bariatric surgery in a subset of patients (insulin conc could not be determined at 30 minutes for all patients), calculated as the product of 1 /fasting insulin and the insulinogenic index (ie, insulin ${ }_{\Delta 0-30} /$ glucose $_{\Delta 0-30}$ ). GLP-1 levels during OMTT was determined by Area Under Curve (AUC), calculated by the trapezoid rule with 0 as baseline. Differences before and after surgery in fasting levels and AUC were analyzed using a paired t-test. Statistical analysis was done by SigmaStat 4.0 (Systat Software, Inc.). Significance level was set to $\mathrm{P}<0.05$.

\section{Results}

\section{Subject characteristics}

The baseline characteristics of the subjects are presented in (Table 1). Including only patients with NGT, surgery did not significantly alter fasting plasma glucose $(5.1 \pm 0.1$ vs. $5.0 \pm 0.1 \mathrm{mM})$. However, mean weight and BMI were significantly different after surgery. Analysis of changes in weight indicated an average weight loss of $20.5 \pm 1.3 \mathrm{~kg}$ at 3 months following bariatric surgery, with a compared to baseline significantly lower mean weight of $91.8 \pm 4.5 \mathrm{~kg}$. Bariatric surgery was also associated with a significant reduction in BMI $(\mathrm{p}=<0.001)$. However, after an average decrease in BMI of $7.2 \pm 0.6 \mathrm{~kg} / \mathrm{m}^{2}$, patients were still obese 3 months after surgery, as indicated by a mean BMI of $34.2 \pm 1.3 \mathrm{~kg} / \mathrm{m}^{2}$.

Table 1: Metabolic characteristics of the subjects at baseline. BMI-Body Mass Index, HOMA-IR-Homeostasis model assessment of insulin resistance.

\begin{tabular}{|c|c|}
\hline & Mean \\
\hline Weight $(\mathrm{kg})$ & $117.7 \pm 13.8$ \\
\hline BMI $\left(\mathrm{kg} / \mathrm{m}^{2}\right)$ & $41.4 \pm 1.3$ \\
\hline Fasting glucose $(\mathrm{mg} / \mathrm{dL})$ & $92 \pm 2$ \\
\hline Fasting insulin $(\mathrm{uIU} / \mathrm{mL})$ & $25.8 \pm 4$ \\
\hline HOMA-IR & $6 \pm 0.9$ \\
\hline
\end{tabular}

\section{Insulin and GLP-1 secretion at fasting}

Fasting plasma insulin and HOMA-IR was significantly reduced after surgery (Figure 1A), where obese subjects included in the study had 6-fold higher fasting insulin going into the study compared to 3 months after bariatric surgery, corresponding to an 80\% HOMA-IR reduction 3 months postoperatively. Fasting GLP-1 levels were 40\% higher in obese subjects before bariatric surgery as compared to 3 months postoperatively $(20.1 \pm 3.5$ vs $12.2 \pm 1.5 \mathrm{pmol} / \mathrm{L}, \mathrm{p}=0.029)$ (Figure 1B).
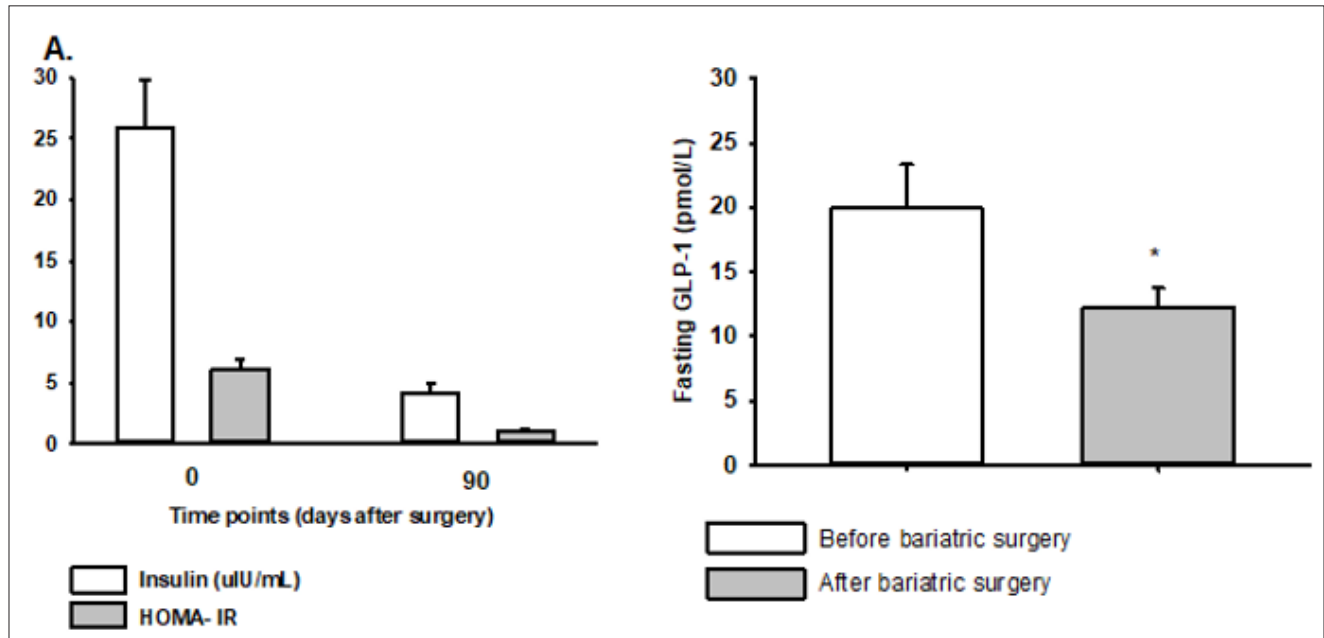

Figure 1: Fasting plasma levels of insulin and HOMA-IR (A), and GLP-1 (B) in obese NGT subjects before and after bariatric surgery, $n=11$. Values are means \pm SEM. *denotes $\mathrm{p}<0.05$ for outcome measures after surgery as compared to before surgery

\section{Insulin and GLP-1 secretion during OMTT}

The peak in postprandial insulin levels was 95\% higher 3 months postoperatively compared to baseline, with peak insulin levels $15 \mathrm{~min}$ into the OMTT as compared to $30 \mathrm{~min}$. The Oral
Disposition Index (DIo), used as a measurement of $\beta$-cell function [33], was significantly increased following bariatric surgery, with $\Delta \mathrm{I} 0-30 / \Delta \mathrm{G} 0-30 \times 1 /$ fasting insulin of $0.67 \pm 0.2$ presurgically and 8.5 \pm 3.5 postsurgically. Postprandial GLP-1 levels increased slowly 
but marginally in obese subjects prior to bariatric surgery. Peak levels $(23 \pm 3 \mathrm{pmol} / \mathrm{L})$ at 30 minutes into the OMTT were not significantly different from the relatively high fasting levels of GLP-1 at baseline (Figure 2A). Three months postoperatively postprandial
GLP-1 levels increased rapidly and peaked (62 \pm 11 pmol/L) 30 minutes into the OMTT in the same subjects, resulting in a $64 \%$ higher peak (Figure 2A). The resulting increase in the Area Under the Curve (AUC) was significant (Figure 2B).

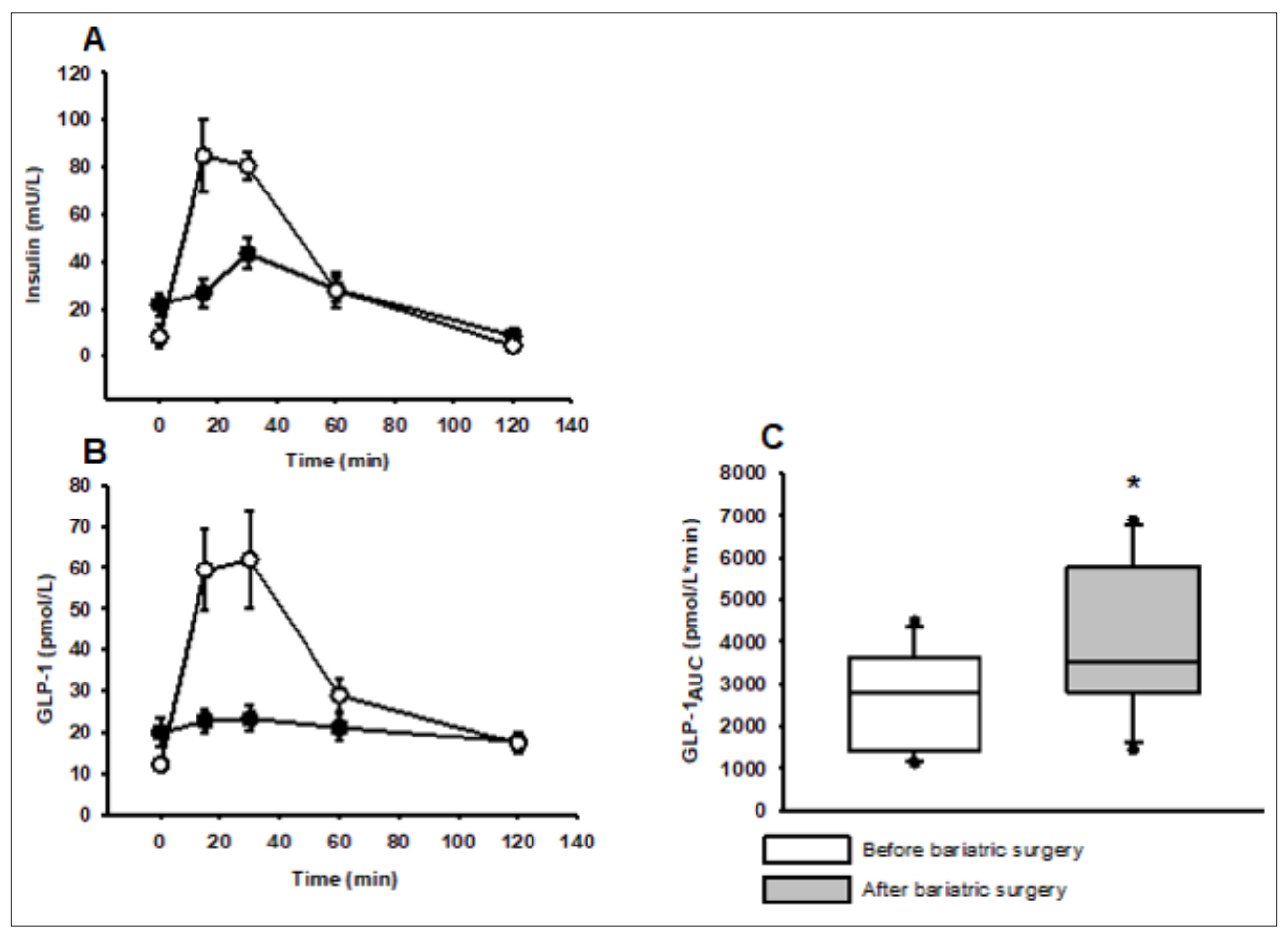

Figure 2: Postprandial GLP-1 plasma levels after bariatric surgery (open circles) and before surgery (closed circles) (A). AUC for plasma GLP-1 (B), $n=11$. Values are means \pm SEM. *denotes $\mathrm{p}<0.05$ for outcome measures after surgery as compared to before surgery

\section{Discussion}

Bariatric surgery can be considered metabolic surgery, with important weight loss-independent effects on metabolism, where similar metabolic outcomes are observed between RYGB and VSG [34]. The degree to which GLP-1 is responsible for the metabolic outcome of bariatric surgery is debated. Importantly though, the effects of GLP-1 receptor agonists (GLP-1RAs), or lack thereof, must not be considered representative of the effects of GLP-1 or its importance in the metabolic outcome of bariatric surgery [35]. Differences in doses and routes of administration are important to consider, where GLP-1 likely acts as a neurotransmitter, while GLP1RAs act on peripheral targets. In this study we demonstrate that baseline fasting GLP-1 levels are decreased in obese NGT women 3 months after bariatric surgery, while postprandial GLP-1 secretion is restored and significantly increased 3 months postoperatively. These findings occur together with reduced insulin resistance, improved beta cell function and increased postprandial insulin secretion 3 months postoperatively. All patients had elevated baseline fasting plasma insulin and HOMA-IR values representing significant insulin resistance ( $>3$ ) despite NGT, indicating insulin hypersecretion. Three months postoperatively, all patients displayed normal insulin sensitivity, with HOMA-IR values ranging between 0.5 and 1.7, despite a mean BMI still indicating obesity.
Beta cell function was significantly improved as assessed by calculation of the DIo. The DIo at baseline agrees well with what has previously been reported for obese subjects; however, DIo 3 months postoperatively indicates beta cell function comparable to that of lean subjects [36]. This drastic effect on DIo may be of considerable importance as DIo predicts risk of incident diabetes at 10 years in nondiabetic subjects, independent of age, sex, BMI, family history of diabetes and baseline fasting and $2 \mathrm{~h}$ glucose concentrations [36]. The present findings are in agreement with reported lower GLP1 responses in obesity regardless of glucose tolerance [37], and decresed fasting GLP-1 associated with weight loss [38]. However, the reported decrease in fasting GLP-1 is in contrast to a recent meta-analysis reporting no significant difference in fasting plasma GLP-1 following bariatric surgery [14]. It needs to be considered though that only three of the studies included attempted to analyze total fasting plasma GLP-1 [7-36] in NGT subjects, and another RCT published demonstrates increased fasting plasma GLP-1 (ref). The discrepancy between results may emphasing the importance of scrutinizing the studies performed in terms of potential age and gender differences as well as surgical procedure.

Further, for the present report plasma GLP-1 levels were analyzed using an ELISA specific for total levels of plasma GLP1 7-36 and the metabolite GLP-1 9-36, avoiding differences in 
degradation as a confounding factor. Whether the higher fasting GLP-1 levels at baseline represent L-cell dysfunction [39] and/or a response to insulin resistance and increased fasting plasma insulin levels [40], or even potential incretin resistance, in obesity remains to be investigated. A possible effect of bariatric surgery involving improved GLP-1 sensitivity/regulation of GLP-1 secretion, may affect other genes, such as the anti-aging gene Sirtuin 1 that regulates beta cell mass [41-44]. It would be of interest to in a larger study also assess potential associations between fasting plasma GLP-1 and Sirtuin 1 gene expression. However, it should be mentioned that although fasting plasma GLP-1 levels in these obese subjects with NGT were reduced 3 months postoperatively, baseline levels were similar to those previously reported for lean nondiabetic subjects [36].

\section{Limitations}

The main weakness of this study is the limited number of patients included. Another weakness is the lack of a lean control group. Further, in this report, we do not discriminate between RYGB and VSG. Although the metabolic outcome of RYGB and VSG is similar, the mechanism are most likely different $[34,45]$. Future studies should focus on carefully delineating intestinal hormone responses between these procedures to facilitate understanding of the mechanisms that underlie metabolic surgical success. The clinical study in which these patients were included will at completion be statistically powered to detect not only differences in gut hormone secretion between RYGB and VSG patients, but also allow for linear regression analysis of associations between beta cell function, insulin resistance, weight loss, BMI etc and gut hormone secretion.

\section{Conclusion}

Despite the relatively low number of patients included, this early report from a larger clinical study presents important and interesting data in terms of reduced fasting GLP-1 in obese subjects with NGT following bariatric surgery. In addition, this report supports increased postprandial GLP-1 secretion in association with reduced insulin resistance and increased beta cell function following bariatric surgery in this patient group.

\section{Acknowledgment}

The authors would like to thank the surgical patients from Södertälje Hospital who were involved in the study for their contribution. This work was supported by the Mats Kleberg foundation and Tore Nilssons foundation.

\section{References}

1. Madsen LR, Baggesen LM, Richelsen B, Thomsen RW (2019) Effect of Roux-en-Y gastric bypass surgery on diabetes remission and complications in individuals with type 2 diabetes: A Danish populationbased matched cohort study. Diabetologia 62(4): 611-620.

2. Schauer PR, Bhatt DL, Kirwan JP, Wolski K, Aminian A, et al. (2017) Bariatric surgery versus intensive medical therapy for diabetes-5-year outcomes. N Engl J Med 376(7): 641-651.

3. Beglinger S, Meyer Gerspach AC, Graf S, Zumsteg U, Drewe J, et al. (2014) Effect of a test meal on meal responses of satiation hormones and their association to insulin resistance in obese adolescents. Obesity (Silver Spring) 22(9): 2047-2052.

4. Verdich C, Toubro S, Buemann B, Lysgard Madsen J, Juul Holst J, et al. (2001) The role of postprandial releases of insulin and incretin hormones in meal-induced satiety--effect of obesity and weight reduction. Int J Obes Relat Metab Disord 25(8): 1206-1214.

5. Vilsboll T, Krarup T, Sonne J, Madsbad S, Volund A, et al. (2003) Incretin secretion in relation to meal size and body weight in healthy subjects and people with type 1 and type 2 diabetes mellitus. J Clin Endocrinol Metab 88(6): 2706-2713.

6. Adam TC, Lejeune MP, Westerterp Plantenga MS (2006) Nutrientstimulated glucagon-like peptide 1 release after body-weight loss and weight maintenance in human subjects. Br J Nutr 95(1): 160-167.

7. Svendsen PF, Jensen FK, Holst JJ, Haugaard SB, Nilas L, et al. (2012) The effect of a very low calorie diet on insulin sensitivity, beta cell function, insulin clearance, incretin hormone secretion, androgen levels and body composition in obese young women. Scand J Clin Lab Invest 72(5): 410419.

8. Peterli R, Steinert RE, Woelnerhanssen B, Peters T, Christoffel Courtin C, et al. (2012) Metabolic and hormonal changes after laparoscopic Rouxen-Y gastric bypass and sleeve gastrectomy: A randomized, prospective trial. Obes Surg 22(5): 740-748.

9. Casajoana A, Pujol J, Garcia A, Elvira J, Virgili N, et al. (2017) Predictive value of gut peptides in T2D remission: Randomized controlled trial comparing metabolic gastric bypass, sleeve gastrectomy and greater curvature plication. Obes Surg 27(9): 2235-2245.

10. Nosso G, Griffo E, Cotugno M, Saldalamacchia G, Lupoli R, et al. (2016) Comparative effects of Roux-en-Y gastric bypass and sleeve gastrectomy on glucose homeostasis and incretin hormones in obese type 2 diabetic patients: A one-year prospective study. Horm Metab Res 48(5): 312-317.

11. Alamuddin N, Vetter ML, Ahima RS, Hesson L, Ritter S, et al. (2017) Changes in fasting and prandial gut and adiposity hormones following vertical sleeve gastrectomy or Roux-en-Y-gastric bypass: An 18-month prospective study. Obes Surg 27(6): 1563-1572.

12. Falken Y, Hellstrom PM, Holst JJ, Naslund E (2011) Changes in glucose homeostasis after Roux-en-Y gastric bypass surgery for obesity at day three, two months, and one year after surgery: Role of gut peptides. J Clin Endocrinol Metab 96(7): 2227-2235.

13. Korner J, Bessler M, Inabnet W, Taveras C, Holst JJ (2007) Exaggerated glucagon-like peptide-1 and blunted glucose-dependent insulinotropic peptide secretion are associated with Roux-en-Y gastric bypass but not adjustable gastric banding. Surg Obes Relat Dis 3(6): 597-601.

14. Jirapinyo P, Jin DX, Qazi T, Mishra N, Thompson CC (2018) A metaanalysis of GLP-1 after Roux-En-Y gastric bypass: Impact of surgical technique and measurement strategy. Obes Surg 28(3): 615-626.

15. Evans S, Pamuklar Z, Rosko J, Mahaney P, Jiang N, et al. (2012) Gastric bypass surgery restores meal stimulation of the anorexigenic gut hormones glucagon-like peptide- 1 and peptide YY independently of caloric restriction. Surg Endosc 26(4): 1086-1094.

16. Gandolfini MP, Coupaye M, Bouaziz E, Dehoux M, Hajage D, et al. (2015) Cardiovascular changes after gastric bypass surgery: Involvement of increased secretions of glucagon-like peptide-1 and brain natriuretic peptide. Obes Surg 25(10): 1933-1939.

17. Korner J, Inabnet W, Febres G, Conwell IM, McMahon DJ, et al. (2009) Prospective study of gut hormone and metabolic changes after adjustable gastric banding and Roux-en-Y gastric bypass. Int J Obes (Lond) 33(7): 786-795.

18. Samat A, Malin SK, Huang H, Schauer PR, Kirwan JP, et al. (2013) Ghrelin suppression is associated with weight loss and insulin action following gastric bypass surgery at 12 months in obese adults with type 2 diabetes. Diabetes Obes Metab 15(10): 963-966. 
19. Umeda LM, Silva EA, Carneiro G, Arasaki CH, Geloneze B, et al. (2011) Early improvement in glycemic control after bariatric surgery and its relationships with insulin, GLP-1, and glucagon secretion in type 2 diabetic patients. Obes Surg 21(7): 896-901.

20. Zhang X, Cheng Z, Xiao Z, Du X, Du J, et al. (2015) Comparison of shortand mid-term efficacy and the mechanisms of gastric bypass surgeries on managing obese and nonobese type 2 diabetes mellitus: A prospective study. Arch Med Res 46(4): 303-309.

21. Breitman I, Saraf N, Kakade M, Yellumahanthi K, White M, et al. (2011) The effects of an amino acid supplement on glucose homeostasis, inflammatory markers, and incretins after laparoscopic gastric bypass. J Am Coll Surg 212(4): 617-625.

22. Chronaiou A, Tsoli M, Kehagias I, Leotsinidis M, Kalfarentzos F, et al. (2012) Lower ghrelin levels and exaggerated postprandial peptideYY, glucagon-like peptide-1, and insulin responses, after gastric fundus resection, in patients undergoing Roux-en-Y gastric bypass: A randomized clinical trial. Obes Surg 22(11): 1761-1770.

23. Lips MA, de Groot GH, van Klinken JB, Aarts E, Berends FJ, et al. (2014) Calorie restriction is a major determinant of the short-term metabolic effects of gastric bypass surgery in obese type 2 diabetic patients. Clin Endocrinol (Oxf) 80(6): 834-842.

24. Morinigo R, Lacy AM, Casamitjana R, Delgado S, Gomis R, et al. (2006) GLP-1 and changes in glucose tolerance following gastric bypass surgery in morbidly obese subjects. Obes Surg 16(12): 1594-1601.

25. Yousseif A, Emmanuel J, Karra E, Millet Q, Elkalaawy M, et al. (2014) Differential effects of laparoscopic sleeve gastrectomy and laparoscopic gastric bypass on appetite, circulating acyl-ghrelin, peptide YY3-36 and active GLP-1 levels in non-diabetic humans. Obes Surg 24(2): 241-252.

26. Borg CM, le Roux CW, Ghatei MA, Bloom SR, Patel AG, et al. (2006) Progressive rise in gut hormone levels after Roux-en-Y gastric bypass suggests gut adaptation and explains altered satiety. Br J Surg 93(2): 210-215.

27. Jorgensen NB, Jacobsen SH, Dirksen C, Bojsen Moller KN, Naver L, et al. (2012) Acute and long-term effects of Roux-en-Y gastric bypass on glucose metabolism in subjects with type 2 diabetes and normal glucose tolerance. Am J Physiol Endocrinol Metab 303(1): E122-E131.

28. Rubino F, Gagner M, Gentileschi P, Kini S, Fukuyama S, et al. (2004) The early effect of the Roux-en-Y gastric bypass on hormones involved in body weight regulation and glucose metabolism. Ann Surg 240(2): 236242.

29. Jacobsen SH, Bojsen Moller KN, Dirksen C, Jorgensen NB, Clausen TR, et al. (2013) Effects of gastric bypass surgery on glucose absorption and metabolism during a mixed meal in glucose-tolerant individuals. Diabetologia 56(10): 2250-2254

30. Dirksen C, Jorgensen NB, Bojsen Moller KN, Kielgast U, Jacobsen SH, et al. (2013) Gut hormones, early dumping and resting energy expenditure in patients with good and poor weight loss response after Roux-en-Y gastric bypass. Int J Obes (Lond) 37(11): 1452-1459.

31. Shah M, Law JH, Micheletto F, Sathananthan M, Dalla Man C, et al. (2014) Contribution of endogenous glucagon-like peptide 1 to glucose metabolism after Roux-en-Y gastric bypass. Diabetes 63(2): 483-493.
32. Svane MS, Bojsen Moller KN, Martinussen C, Dirksen C, Madsen JL, et al. (2019) Postprandial nutrient handling and gastrointestinal hormone secretion after Roux-en-Y gastric bypass vs sleeve gastrectomy. Gastroenterology 156(6): 1627-1641.

33. Utzschneider KM, Prigeon RL, Faulenbach MV, Tong J, Carr DB, et al. (2009) Oral disposition index predicts the development of future diabetes above and beyond fasting and 2-h glucose levels. Diabetes Care 32(2): 335-341.

34. Hutch CR, Sandoval D (2017) The role of GLP-1 in the metabolic success of bariatric surgery. Endocrinology 158(12): 4139-4151.

35. Michels E (1970) The 1970 presidential address. Phys Ther 50(11): 1579-1590.

36. Manell H, Staaf J, Manukyan L, Kristinsson H, Cen J, et al. (2016) Altered plasma levels of glucagon, GLP-1 and glicentin during OGTT in adolescents with obesity and type 2 diabetes. J Clin Endocrinol Metab 101(3): 1181-1189.

37. Faerch K, Torekov SS, Vistisen D, Johansen NB, Witte DR, et al. (2015) GLP-1 response to oral glucose is reduced in prediabetes, screendetected type 2 diabetes, and obesity and influenced by sex: The ADDITION-PRO study. Diabetes 64(7): 2513-2525.

38. Reinehr T, de Sousa G, Roth CL (2007) Fasting glucagon-like peptide-1 and its relation to insulin in obese children before and after weight loss. J Pediatr Gastroenterol Nutr 44(5): 608-612.

39. Ntika S, Thombare K, Aryapoor M, Kristinsson H, Bergsten P, et al. (2018) Oleate increase neutral lipid accumulation, cellular respiration and rescues palmitate-exposed GLP-1 secreting cells by reducing ceramideinduced ROS. Biochimie 59: 23-35.

40. Kappe C, Zhang Q, Holst JJ, Nystrom T, Sjoholm A (2013) Evidence for paracrine/autocrine regulation of GLP-1-producing cells. Am J Physiol Cell Physiol 305(10): C1041- C1049.

41. Wang Y, Wang DS, Cheng YS, Jia BL, Yu G, et al. (2018) Expression of MicroRNA-448 and SIRT1 and prognosis of obese type 2 diabetic mellitus patients after laparoscopic bariatric surgery. Cell Physiol Biochem 45(3): 935-950.

42. Mohammadi Farsani T, Mohammadi Farsani G, Kabir A (2019) Gene expression changings after bariatric surgery in morbid obese patients and type 2 diabetes remission. Obes Surg 29(2): 649-650.

43. Bastien Dionne PO, Valenti L, Kon N, Gu W, Buteau J (2011) Glucagon-like peptide 1 inhibits the sirtuin deacetylase SirT1 to stimulate pancreatic beta-cell mass expansion. Diabetes 60(12): 3217-3222.

44. Desai T, Koulajian K, Ivovic A, Breen DM, Luu L, et al. (2019) Pharmacologic or genetic activation of SIRT1 attenuates the fat-induced decrease in beta-cell function in vivo. Nutr Diabetes 9(1): 11.

45. Amouyal C, Andreelli F (2016) Increasing GLP-1 circulating levels by bariatric surgery or by GLP-1 receptor agonists therapy: Why are the clinical consequences so different? J Diabetes Res 2016: 5908656. 